Article

\title{
Aspirin Use among Adults with Cardiovascular Disease in the United States: Implications for an Intervention Approach
}

\author{
Benjamin E. Ansa $1, * \mathbb{B}$, Zachary Hoffman ${ }^{2}$, Nicollette Lewis ${ }^{3}$, Cassandra Savoy ${ }^{4}$, \\ Angela Hickson ${ }^{5}$, Rebecca Stone ${ }^{1}\left(\mathbb{D}\right.$ and Tara Johnson ${ }^{6}$ \\ 1 Institute of Public \& Preventive Health, Augusta University, Augusta, GA 30912, USA; bstone@augusta.edu \\ 2 Master of Science in Experimental Psychology Program, Augusta University, Augusta, GA 30912, USA; \\ zhoffma1@augusta.edu \\ 3 Medical College of Georgia, Augusta University, Augusta, GA 30912, USA; nilewis@augusta.edu \\ 4 Burke Medical Center of Waynesboro, Waynesboro, GA 30830, USA; swtcheeks_1966@yahoo.com \\ 5 Master of Public Health Program, Augusta University, Augusta, GA 30912, USA; ahickso3@augusta.edu \\ 6 Belvedere Elementary School, Aiken County Public School District, N. Augusta, SC 29841, USA; \\ tjohnson3@acpsd.net \\ * Correspondence: bansa@augusta.edu; Tel.: +1-706-721-6141
}

Received: 30 January 2019; Accepted: 18 February 2019; Published: 20 February 2019

\begin{abstract}
Cardiovascular disease (CVD) is a major underlying cause of death, with high economic burden in most countries, including the United States. Lifestyle modifications and the use of antiplatelet therapy, such as aspirin, can contribute significantly to secondary prevention of CVD in adults. This study examined the prevalence and associated factors of aspirin use for the secondary prevention of angina pectoris, myocardial infarction (MI), and cerebrovascular disease (stroke) in a sample of American adults. The 2015 Behavioral Risk Factor Surveillance System (BRFSS) dataset was analyzed for this cross-sectional study. Almost $16 \%$ of the study population $(N=441,456)$ had angina, MI, or stroke. Weighted percentages of respondents with angina, MI, and stroke were $4 \%$, $4.3 \%$, and $3 \%$, respectively. Overall, weighted prevalence of daily (or every other day) aspirin use was about $65 \%, 71 \%$, and $57 \%$ among respondents with angina, $\mathrm{MI}$, and stroke, respectively. Factors that were significantly associated with aspirin use included male sex, more than high school education, high blood pressure, diabetes, and less than excellent general health. There were existing differences among individuals with CVD based on diagnosis, demographic and socioeconomic status in the use of aspirin for secondary prevention. Resources for promoting aspirin use should be directed toward groups with lower utilization.
\end{abstract}

Keywords: aspirin; cardiovascular disease; angina; myocardial infarction; stroke; prevalence; United States; BRFSS

\section{Introduction}

Cardiovascular disease (CVD) is a term for a number of disorders affecting the heart and blood vessels, which includes coronary heart disease (CHD), myocardial infarction, cerebrovascular disease, peripheral arterial disease, rheumatic and congenital heart diseases, and venous thromboembolism [1-3]. CVD has been listed as the major underlying cause of death in most countries. Globally, more than 17.3 million deaths due to CVD were estimated in 2013, accounting for $31 \%$ of all global mortality. This number is expected to grow to more than 23.6 million deaths by 2030 [1,2]. More than 92 million American adults have a diagnosis of CVD, with nearly 801,000 mortality cases, representing 1 out of every 3 deaths [4]. The direct and indirect costs of CVD and 
stroke in America are estimated to total more than $\$ 316$ billion; this includes both health expenditures and lost productivity [4]. In 2010, the estimated global cost of CVD was $\$ 863$ billion, and it is estimated to rise to $\$ 1044$ billion by 2030 [4].

The risk of CVD can be reduced through lifestyle modifications, such as engaging in recommended physical activity; consuming healthy diets low in sugars and saturated fat and high in vegetables, fruits, and whole grains; avoiding smoking; reducing alcohol intake; and maintaining a healthy weight $[5,6]$. Antiplatelet therapy with agents such as aspirin is a major contributor to secondary prevention of CVD [2]. The cardioprotective benefits of aspirin result from its ability to cause the irreversible acetylation of cyclooxygenase (COX-1 enzyme) in platelets, thereby inhibiting the formation of prostaglandins and thromboxane A2, two potent promoters of platelet aggregation and vasoconstriction [7-10]. These findings are achieved by daily doses of $75 \mathrm{mg}$ (and higher) or perhaps doses as low as $30 \mathrm{mg}$. Low-dose aspirin regimens ( $\geq 30 \mathrm{mg} /$ day) can effectively suppress platelet aggregation without affecting important endothelial cell functions $[8,11]$.

The U.S. Preventive Services Task Force (USPSTF) and other health-related agencies recommend aspirin use for the primary and secondary prevention of CVD in adults based on recognized risks, such as older age, male sex, race/ethnicity, abnormal lipid levels, high blood pressure, diabetes, and smoking status [12-16]. Although the role of aspirin for primary CVD prevention remains controversial [8], aspirin use for secondary prevention of CVD causes a significant reduction in nonfatal reinfarction, stroke, 5-week vascular mortality, and all-cause mortality [17]. Low-dose aspirin reduces the risk of vascular events (myocardial infarction (MI), stroke, and vascular death) in patients who have experienced an MI or a stroke or who are at high risk of vascular disease by Framingham risk score [18].

Several studies have examined the prevalence of aspirin use for the prevention of CVD as a group of disorders, but very few have examined its use for the prevention of specific disorders grouped under CVD. This study has three aims: (1) to examine the prevalence of CVD (angina pectoris, myocardial infarction, and cerebrovascular disease (stroke)) in a sample of American adults; (2) to examine the use of aspirin for the secondary prevention of angina pectoris, myocardial infarction, and cerebrovascular disease (stroke); and (3) to identify the factors associated with aspirin use among adults with CVD.

\section{Materials and Methods}

\subsection{Study Design}

The 2015 Behavioral Risk Factor Surveillance System (BRFSS) dataset was analyzed for this cross-sectional study.

\subsection{Data Source, Study Participants, and Sampling}

The BRFSS is a nationally representative cross-sectional survey that collects data on U.S. residents in all 50 states, the District of Columbia, and three U.S. territories regarding their health-related risk behaviors, chronic health conditions, and use of preventive services [19]. The BRFSS is a state-based, random-digit-dialed telephone survey of the noninstitutionalized U.S. civilian population aged 18 years or older $[19,20]$. Surveys are conducted through phone interviews (landline and cellphone), and more than 400,000 adult interviews are conducted each year, making it the largest continuously conducted health survey system in the world and a useful tool for addressing and developing health promotion activities [19,21]. Although conducted in different time periods, the surveys used identical methods for recruitment. Response rates for BRFSS were calculated using standards set by the American Association of Public Opinion Research (AAPOR) Response Rate Formula 4 [22]. The median survey response rate (\%) for all states and Washington, DC, was 47.2 in 2015 and ranged from 33.9 to 61.1 [23]. 


\subsection{Measures}

Respondents were categorized under sociodemographic variables of sex (male or female); age in years (18-34, 35-44, 45-64, or $\geq 65$ ); race (non-Hispanic (NH) white, NH black, Hispanic, other, or unknown); education (<high school, high school/General Educational Development (GED), $>$ high school); annual income in United States dollar (USD $(<\$ 25,000, \$ 25,000-\$ 50,000$ and $>\$ 50,000)$ ); marital status (single relationship (divorced, widowed, separated, never married) and couple relationship (married or a member of an unmarried couple)); and healthcare coverage (yes/no). Smoking status was categorized as 1 every day, 2 some days, and not at all. Clinical characteristics included general health (poor/fair, good/very good, excellent); high blood pressure (yes/no); diabetes (yes/no); cholesterol (yes/no); and body mass index (BMI) (underweight, normal weight, overweight, obese). The outcome variables were participants' response to the following questions: "Do you take aspirin daily or every other day?" (yes/no); "Have you ever been told by a doctor, nurse, or other health professional that you had a heart attack, also called a myocardial infarction?"(yes/no); "Have you ever been told by a doctor, nurse, or other health professional that you had angina or coronary heart disease?" (yes/no); and "Have you ever been told by a doctor, nurse, or other health professional that you had a stroke?" (yes/no).

\subsection{Statistical Analyses}

Descriptive statistics of sociodemographic and clinical variables related to CVD status and aspirin use were generated for 2015 using frequencies and proportions. Data were weighted using the iterative proportional fitting weighting method (i.e., raking) to adjust for noncoverage, nonresponse, and for differences between sample and population characteristics [24]. Raking is a method for adjusting the sampling weights of the sample data based on known population characteristics. Weighting is used to adjust the results of a study to bring them more in line with what is known about a population [25]. Weighted percentages of respondents who reported having CVD and use aspirin were calculated for each variable category. A weight is computed for every respondent in a sample, and it is computed by dividing the correct proportion by the observed proportion [25]. Logistic regression analyses were conducted to examine the association between sociodemographic variables and aspirin use among CVD respondents. Data were adjusted for sociodemographic and clinical status. Odds ratios and related $95 \%$ confidence intervals were derived from regression analysis. Chi-square test was used to obtain $p$-values. The significance level was set at $p<0.05$, and all tests were two-sided. Unweighted counts, weighted percentages, and logistic regression analyses were performed using the IBM SPSS version 25 (IBM Corp., Armonk, NY, USA) [26].

\subsection{Ethical Considerations}

BRFSS datasets are publicly accessible and do not contain personally identifiable information. The Centers for Disease Control and Prevention (CDC) ensures that the process of data collection and release are governed by appropriate rules, regulations, and legislative authorizations [27].

\section{Results}

\subsection{Sociodemographic and Clinical Characteristics of the Study Population}

In the 2015 BRFSS database, a total of 441,456 adults responded to the question "Have you ever been told by a doctor, nurse, or other health professional that you had a heart attack (MI), angina, or stroke?" The respondents were $\geq 18$ years old, predominantly female $(57.7 \%)$, NH white $(76.1 \%)$, between 45 and 64 years old (38.6\%), with greater than high school education (63.9\%), in a couple relationship (55.7\%), with an annual income $>\$ 50,000$ (39.3\%), and had healthcare coverage $(92.3 \%)$ (Table 1). A majority reported good/very good general health $(63.9 \%)$, no high blood pressure (59.3\%), no diabetes $(84.3 \%)$, and no high blood cholesterol level (49.6\%). For BMI and smoking status, 
there were 405,058 and 184,193 respondents, respectively. A majority of respondents were overweight $(36.3 \%)$ and did not smoke at all $(66.4 \%)$.

About $15.6 \%(N=69,031)$ of respondents had CVD. More men reported having angina $(53.3 \%)$ and MI (56.9\%), while more women reported having stroke (58.3\%). The majority of respondents $(>60 \%)$ that reported a diagnosis of angina, MI, or stroke were 65 years and older. The results of the descriptive analyses of sociodemographic and clinical categories by CVD status were statistically significant.

Table 1. Sociodemographic and clinical characteristics of the study population by CVD Status: BRFSS 2015 data.

\begin{tabular}{|c|c|c|c|c|c|}
\hline \multirow[b]{2}{*}{ Variables } & \multirow{2}{*}{$\begin{array}{c}\text { Total } \\
N=441,456(\%)\end{array}$} & \multirow{2}{*}{$\begin{array}{c}\text { No CVD } \\
N=372,425(\%)\end{array}$} & \multicolumn{3}{|c|}{$\begin{array}{c}\text { Cardiovascular Disease (CVD) } \\
\qquad N=69,031(\%)\end{array}$} \\
\hline & & & $\begin{array}{c}\text { Angina } \\
N=25,290(\%)\end{array}$ & $\begin{array}{c}\text { Myocardial } \\
\text { Infarction } \\
N=25,472(\%)\end{array}$ & $\begin{array}{c}\text { Stroke } \\
N=18,269(\%)\end{array}$ \\
\hline \multicolumn{6}{|l|}{ Sex } \\
\hline Male & $186,938(42.3)$ & $151,350(40.6)$ & $13,490(53.3)$ & $14,484(56.9)$ & $7,614(41.7) *$ \\
\hline Female & $254,518(57.7)$ & $221,075(59.4)$ & $11,800(46.7)$ & $10,988(43.1)$ & $10,655(58.3)$ * \\
\hline \multicolumn{6}{|l|}{ Age (years) } \\
\hline $18-34$ & $66,915(15.2)$ & $65,988(17.7)$ & $274(1.1)$ & $313(1.2)$ & $340(1.9)$ \\
\hline $35-44$ & $50,819(11.5)$ & $49,033(13.2)$ & 487 (1.9) & $646(2.5)$ & $653(3.6)$ \\
\hline $45-64$ & $170,513(38.6)$ & $149,048(40.0)$ & 7618 (30.1) & 7939 (31.2) & $5908(32.3)$ \\
\hline $65+$ & $153,209(34.7)$ & $108,356(29.1)$ & $16,911(66.9)$ & $16,574(65.1)$ & $11,368(62.2)$ \\
\hline \multicolumn{6}{|l|}{ Race } \\
\hline NH white & $336,066(76.1)$ & $281,672(75.6)$ & $20,430(80.8)$ & $20,232(79.4)$ & $13,732(75.2)$ \\
\hline NH black & $34,346(7.8)$ & $28,616(7.7)$ & $1758(7.0)$ & $1872(7.4)$ & $2100(11.5)$ \\
\hline Hispanic & $35,795(8.1)$ & $32,194(8.6)$ & $1354(5.4)$ & $1384(5.4)$ & $863(4.7)$ \\
\hline Other & $27,812(6.3)$ & $23,850(6.4)$ & $1301(5.0)$ & $1474(5.8)$ & $1187(6.5)$ \\
\hline Unknown & $7437(1.7)$ & $6093(1.6)$ & $447(1.8)$ & $510(2.0)$ & $387(2.1)$ \\
\hline \multicolumn{6}{|l|}{ Education } \\
\hline$<$ High school & $34,259(7.8)$ & $24,994(6.7)$ & $2930(11.6)$ & $3622(14.2)$ & $2713(14.9)$ \\
\hline High school & $123,227(27.9)$ & $100,225(26.9)$ & 8066 (31.9) & $8790(34.5)$ & 6146 (33.6) \\
\hline$>$ High school & $282,159(63.9)$ & $245,666(66.0)$ & $14,204(56.1)$ & $12,951(50.8)$ & $9338(51.1)$ \\
\hline Don't know/Refused & $1811(0.4)$ & $1540(0.4)$ & $90(0.4)$ & $109(0.4)$ & $72(0.4)$ \\
\hline \multicolumn{6}{|l|}{ Income } \\
\hline$<\$ 25 \mathrm{~K}$ & $97,222(22.0)$ & $72,871(19.6)$ & $8190(32.4)$ & 9037 (35.5) & $7124(39.0)$ \\
\hline $25 \mathrm{~K}-50 \mathrm{~K}$ & $91,287(20.7)$ & $75,773(20.3)$ & $5817(23.0)$ & $5815(22.8)$ & $3882(21.2)$ \\
\hline$>50 \mathrm{~K}$ & $173,442(39.3)$ & $157,446(42.3)$ & $6755(26.7)$ & $5771(22.7)$ & $3470(19.0)$ \\
\hline Don't know/Refused & 79,505 (18.0) & $66,335(17.8)$ & 4528 (17.9) & $4849(19.0)$ & $3793(20.8)$ \\
\hline \multicolumn{6}{|l|}{ Marital status } \\
\hline Single relationship & $192,523(43.6)$ & $156,520(42.1)$ & $12,391(49.0)$ & 13,167 (51.7) & 10,445 (57.2) \\
\hline Couple relationship & $245,837(55.7)$ & $213,125(57.2)$ & $12,797(50.6)$ & $12,194(47.9)$ & 7721 (42.3) \\
\hline Don't know/Refused & $3096(0.7)$ & $2780(0.7)$ & $102(0.4)$ & $111(0.4)$ & $103(0.6)$ \\
\hline \multicolumn{6}{|l|}{ Health coverage } \\
\hline Yes & $407,556(92.3)$ & 341,549 (91.7) & $24,377(96.4)$ & $24,275(95.3)$ & $17,355(95.0)$ \\
\hline No & $32,060(7.3)$ & $29,233(7.8)$ & $861(3.4)$ & $1116(4.4)$ & $850(4.7)$ \\
\hline $\begin{array}{l}\text { Don't } \\
\text { Know/Refused }\end{array}$ & $1840(0.4)$ & $1643(0.5)$ & $52(0.2)$ & $81(0.3)$ & $64(0.3)$ \\
\hline \multicolumn{6}{|l|}{ General health } \\
\hline Poor/Fair & 82,137 (18.6) & $47,898(12.9)$ & $12,612(49.9)$ & $12,527(49.2)$ & $9100(49.8)$ \\
\hline Good/Very good & $282,040(63.9)$ & $250,317(67.2)$ & $11,708(46.3)$ & $11,756(46.1)$ & 8259 (45.2) \\
\hline Excellent & $76,032(17.2)$ & $73,327(19.7)$ & $867(3.4)$ & $1044(4.1)$ & $794(4.3)$ \\
\hline Don't know/Refused & $1247(0.3)$ & $883(0.2)$ & $103(0.4)$ & $145(0.6)$ & $116(0.7)$ \\
\hline \multicolumn{6}{|l|}{ High blood pressure } \\
\hline Yes & $178,188(40.4)$ & $126,451(34.0)$ & 19,517 (77.2) & $18,818(73.9)$ & $13,402(73.4)$ \\
\hline No & $261,829(59.3)$ & $245,402(65.8)$ & $5490(21.7)$ & $6319(24.8)$ & 4618 (25.3) \\
\hline Others/Don't know & $1439(0.3)$ & $572(0.2)$ & $283(1.1)$ & 335 (1.3) & 249 (1.3) \\
\hline
\end{tabular}


Table 1. Cont.

\begin{tabular}{|c|c|c|c|c|c|}
\hline \multirow[b]{2}{*}{ Variables } & \multirow{2}{*}{$\begin{array}{c}\text { Total } \\
N=441,456(\%)\end{array}$} & \multirow{2}{*}{$\begin{array}{c}\text { No CVD } \\
N=372,425(\%)\end{array}$} & \multicolumn{3}{|c|}{$\begin{array}{c}\text { Cardiovascular Disease (CVD) } \\
\qquad N=69,031(\%)\end{array}$} \\
\hline & & & $\begin{array}{c}\text { Angina } \\
N=25,290(\%)\end{array}$ & $\begin{array}{c}\text { Myocardial } \\
\text { Infarction } \\
N=25,472(\%)\end{array}$ & $\begin{array}{c}\text { Stroke } \\
N=18,269(\%)\end{array}$ \\
\hline \multicolumn{6}{|l|}{ Diabetes } \\
\hline Yes & $57,256(13.0)$ & $34,630(9.3)$ & 8541 (33.8) & $8526(33.5)$ & $5559(30.4)$ \\
\hline No & $372,104(84.3)$ & $328,019(88.1)$ & $15,901(62.9)$ & $16,113(63.2)$ & $12,071(66.1)$ \\
\hline Others/Don't know & $12,096(2.7)$ & $9776(2.6)$ & $848(3.3)$ & $833(3.3)$ & $639(3.5)$ \\
\hline Cholesterol & $N=441,456$ & $N=375,195$ & $N=24,623$ & $N=24,431$ & $N=17,207$ \\
\hline Yes & $159,970(36.2)$ & $114,752(30.6)$ & $17,754(72.1)$ & $16,637(68.1)$ & $10,827(62.9)$ \\
\hline No & $218,771(49.6)$ & $198,513(52.9)$ & $6628(26.9)$ & 7493 (30.7) & $6137(35.7)$ \\
\hline Don't know/Refused & $62,715(14.2)$ & $61,930(16.5)$ & $241(1.0)$ & $301(1.2)$ & $243(1.4)$ \\
\hline Smoking status & $\mathrm{N}=184,193$ & $N=143,474$ & $N=14,770$ & $N=15,783$ & $N=10,166$ \\
\hline 1 every day & $43,583(23.7)$ & $35,285(24.6)$ & $2479(16.8)$ & $3403(21.6)$ & $2416(23.8)$ \\
\hline 2 some days & $17,998(9.8)$ & $14,599(10.2)$ & $1,058(7.2)$ & $1,301(8.2)$ & $1040(10.2)$ \\
\hline Not at all & $122,277(66.4)$ & $93,348(65.1)$ & $11,197(75.8)$ & $11,035(69.9)$ & $6697(65.9)$ \\
\hline Don't know/Refused & $335(0.2)$ & $242(0.1)$ & $36(0.2)$ & $44(0.3)$ & $13(0.1)$ \\
\hline BMI & $N=405,058$ & $N=339,863$ & $N=23,959$ & $N=24,103$ & $N=17,133$ \\
\hline Underweight & $6721(1.7)$ & $5570(1.6)$ & $335(1.4)$ & 419 (1.7) & $397(2.3)$ \\
\hline Normal weight & $131,409(32.4)$ & $115,264(33.9)$ & $5534(23.1)$ & $5850(24.3)$ & $4761(27.8)$ \\
\hline Overweight & $147,004(36.3)$ & $122,928(36.2)$ & 8967 (37.4) & $9020(37.4)$ & 6089 (35.5) \\
\hline Obese & $119,924(29.6)$ & $96,101(28.3)$ & $9123(38.1)$ & 8814 (36.6) & $5886(34.4)$ \\
\hline
\end{tabular}

BRFSS: Behavioral Risk Factor Surveillance System, NH: non-Hispanic. ${ }^{*}$ All $p$-values $<0.0001$, except for sex vs. stroke $(p$-value $=0.007)$.

\subsection{Weighted Percentages of Respondents with Cardiovascular Disease}

The prevalence of angina and MI was higher than that of stroke. The prevalence of overall angina, MI, and stroke were $4.0 \%, 4.3 \%$, and 3.0\%, respectively (Table 2). Men had higher prevalence (between $4 \%$ and $6 \%$ ) of angina and MI, while women had higher prevalence of stroke (3.1\%). CVD prevalence increased with age, and respondents 65 years and older had the highest prevalence. Angina $(4.7 \%)$ and $\mathrm{MI}(4.8 \%)$ were reported more among $\mathrm{NH}$ white, while $\mathrm{NH}$ black reported more cases of stroke $(4.1 \%)$. Prevalence of CVD decreased with increasing levels of education and income. Also, more cases of CVD were reported among individuals with high blood pressure, high blood cholesterol, and diabetes. Obese and overweight respondents and those reporting poor/fair general health had higher proportions of CVD.

Table 2. Weighted percentages of respondents with cardiovascular disease: BRFSS 2015.

\begin{tabular}{|c|c|c|c|c|}
\hline \multirow[b]{2}{*}{ Variables } & \multicolumn{3}{|c|}{ Cardiovascular Disease } & \multirow[b]{2}{*}{$p$-Value } \\
\hline & $\begin{array}{c}\text { Angina Weighted } \\
N(\%)\end{array}$ & $\begin{array}{l}\text { Myocardial Infarction } \\
\text { Weighted } N(\%)\end{array}$ & $\begin{array}{c}\text { Stroke Weighted } \\
N(\%)\end{array}$ & \\
\hline Overall & $10,154,549(4.0)$ & $10,691,710(4.3)$ & $7,609,840(3.0)$ & \\
\hline Sex & & & & $<0.0001$ \\
\hline Male & $5,883,465(4.8)$ & $6,741,115(5.5)$ & $3,605,072(2.9)$ & \\
\hline Female & $4,271,084(3.3)$ & $3,950,595(3.1)$ & $4,004,768(3.1)$ & \\
\hline Age & & & & $<0.0001$ \\
\hline $18-34$ & $290,818(0.4)$ & $340,991(0.5)$ & $360,840(0.5)$ & \\
\hline $35-44$ & $433,773(1.1)$ & $590,022(1.4)$ & $526,960(1.3)$ & \\
\hline $45-64$ & $3,777,164(4.4)$ & $4,134,347(4.9)$ & $2,971,101(3.5)$ & \\
\hline $65+$ & $5,652,794(11.5)$ & $5,626,350(11.4)$ & $3,750,939(7.6)$ & \\
\hline
\end{tabular}


Table 2. Cont.

\begin{tabular}{|c|c|c|c|c|}
\hline \multirow[b]{2}{*}{ Variables } & \multicolumn{3}{|c|}{ Cardiovascular Disease } & \multirow[b]{2}{*}{$p$-Value } \\
\hline & $\begin{array}{c}\text { Angina Weighted } \\
\qquad(\%)\end{array}$ & $\begin{array}{l}\text { Myocardial Infarction } \\
\text { Weighted N (\%) }\end{array}$ & $\begin{array}{c}\text { Stroke Weighted } \\
\qquad N(\%)\end{array}$ & \\
\hline Race & & & & $<0.0001$ \\
\hline NH white & $7,342,743(4.7)$ & $7,568,147(4.8)$ & $5,049,650(3.2)$ & \\
\hline NH black & $1,008,762(3.5)$ & $1,117,769(3.9)$ & $1,190,126(4.1)$ & \\
\hline Hispanic & $1,039,482(2.5)$ & $1,160,996(2.8)$ & $732,644(1.8)$ & \\
\hline Other & $604,787(3.1)$ & $636,153(3.2)$ & $480,468(2.4)$ & \\
\hline Unknown & $158,775(3.8)$ & $208,644(4.9)$ & $156,951(3.7)$ & \\
\hline Education & & & & $<0.0001$ \\
\hline$<$ High school & $2,078,745(5.8)$ & $2,626,453(7.3)$ & $1,873,946(5.2)$ & \\
\hline High school & $3,133,312(4.4)$ & $3,481,348(4.9)$ & $2,429,170(3.4)$ & \\
\hline >High school & $4,917,798(3.4)$ & $4,543,369(3.2)$ & $3,279,577(2.3)$ & \\
\hline Income & & & & $<0.0001$ \\
\hline$<\$ 25 \mathrm{~K}$ & $3,346,520(5.6)$ & $3,945,885(6.6)$ & $3,055,341(5.1)$ & \\
\hline$\$ 25 \mathrm{~K}-\$ 50 \mathrm{~K}$ & $2,375,159(4.7)$ & $2,354,763(4.7)$ & $1,599,694(3.2)$ & \\
\hline$>\$ 50 \mathrm{~K}$ & $2,709,241(2.8)$ & $2,405,748(2.5)$ & $1,419,673(1.5)$ & \\
\hline Marital status & & & & $<0.0001$ \\
\hline Single relationship & $4,479,142(4.0)$ & $5,118,891(4.6)$ & $3,984,256(3.6)$ & \\
\hline Couple relationship & $5,631,915(4.1)$ & $5,525,284(4.0)$ & $3,582,067(2.6)$ & \\
\hline High blood pressure & & & & $<0.0001$ \\
\hline Yes & $7,718,064(9.6)$ & $7,745,099(9.7)$ & $5,478,837(6.8)$ & \\
\hline No & $2,344,322(1.4)$ & $2,812,256(1.7)$ & $2,037,462(1.2)$ & \\
\hline Smoking & & & & $<0.0001$ \\
\hline 1 every day & $1,112,518(4.1)$ & $1,640,283(6.0)$ & $1,188,411(4.3)$ & \\
\hline 2 some days & $532,100(4.3)$ & $640,950(5.1)$ & $490,845(3.9)$ & \\
\hline Not at all & $4,325,414(7.4)$ & $4,373,889(7.5)$ & $2,606,681(4.5)$ & \\
\hline Cholesterol & & & & $<0.0001$ \\
\hline Yes & $6,983,293(9.7)$ & $6,807,803(9.5)$ & $4,343,065(6.0)$ & \\
\hline No & $2,707,512(2.2)$ & $3,138,353(2.5)$ & $2,625,566(2.1)$ & \\
\hline Diabetes & & & & $<0.0001$ \\
\hline Yes & $3,516,016(13.3)$ & $3,656,625$ (13.9) & $2,286,880(8.7)$ & \\
\hline No & $6,246,247(2.9)$ & $6,657,234(3.1)$ & $5,027,487(2.3)$ & \\
\hline BMI & & & & $<0.0001$ \\
\hline Underweight & $139,326(3.4)$ & $166,135(4.0)$ & $156,285(3.8)$ & \\
\hline Normal weight & $2,145,210(2.8)$ & $2,343,929(3.0)$ & $1,952,532(2.5)$ & \\
\hline Overweight & $3,519,681(4.3)$ & $3,741,929(4.6)$ & $2,509,450(3.1)$ & \\
\hline Obese & $3,791,843(5.7)$ & $3,853,416(5.8)$ & $2,469,480(3.7)$ & \\
\hline General health & & & & $<0.0001$ \\
\hline Poor/Fair & $5,220,703$ (11.8) & $5,483,745(12.4)$ & $3,921,665(8.8)$ & \\
\hline Good/Very good & $4,505,342(2.8)$ & $4,668,229(2.9)$ & $3,261,927(2.0)$ & \\
\hline Excellent & $387,613(0.8)$ & $481,190(1.0)$ & $381,046(0.8)$ & \\
\hline
\end{tabular}

3.3. Weighted Percentages of Respondents with Cardiovascular Disease that Take Aspirin Daily or Every Other Day

Overall, aspirin use was higher among respondents with MI (71.1\%) than among those with angina (64.8\%) and stroke (57.3\%) (Table 3). Aspirin use was also higher among respondents that were males, $\geq 65$ years, with more than high school education, and in a couple relationship. A majority of aspirin users with angina were $\mathrm{NH}$ white $(75.3 \%)$, and a majority of aspirin users with stroke were $\mathrm{NH}$ black $(69.8 \%)$. Also, aspirin use was highest among those with angina and MI earning annual income $>\$ 50,000$ USD $(>82 \%)$, while aspirin use was highest among those with stroke earning annual income of $\$ 25,000-\$ 50,000$ USD (64.8\%). Most respondents with the three forms of CVD that were aspirin 
users either had high blood pressure $(>63 \%)$ or diabetes $(>71 \%)$. The least reported use of aspirin was among individuals with angina that were underweight (31.7\%), with MI and stroke that had normal weight, and with the three forms of CVD that had excellent general health.

Table 3. Weighted percentages of respondents with cardiovascular disease that take aspirin daily or every other day: BRFSS 2015.

\begin{tabular}{|c|c|c|c|c|}
\hline \multirow[b]{2}{*}{ Variables } & \multicolumn{3}{|c|}{ Aspirin Use } & \multirow[b]{2}{*}{$p$-Value } \\
\hline & $\begin{array}{l}\text { Angina } \\
N(\%)\end{array}$ & $\begin{array}{c}\text { Myocardial Infarction } \\
\text { N (\%) }\end{array}$ & $\begin{array}{l}\text { Stroke } \\
N(\%)\end{array}$ & \\
\hline Overall & $406,221(64.8)$ & $400,916(71.1)$ & 236,207 (57.3) & \\
\hline Sex & & & & $<0.0001$ \\
\hline Male & $239,240(71.3)$ & $258,053(72.2)$ & $124,831(60.1)$ & \\
\hline Female & $166,981(57.4)$ & $142,864(72.0)$ & $111,376(54.4)$ & \\
\hline Age & & & & $<0.0001$ \\
\hline $18-34$ & $6064(26.7)$ & 3114 (18.4) & $2350(23.8)$ & \\
\hline $35-44$ & $18,835(45.3)$ & 9598 (50.3) & $18,111(38.6)$ & \\
\hline $45-64$ & $158,054(62.8)$ & $177,984(72.9)$ & $96,543(56.8)$ & \\
\hline $65+$ & 223,268 (71.9) & $210,220(76.2)$ & $119,203(64.2)$ & \\
\hline Race & & & & $<0.0001$ \\
\hline NH white & $253,540(75.3)$ & $259,428(79.1)$ & $147,733(58.5)$ & \\
\hline NH black & $40,408(68.7)$ & $52,729(79.8)$ & $46,027(69.8)$ & \\
\hline Hispanic & $12,801(56.0)$ & $69,728(51.7)$ & $32,928(49.4)$ & \\
\hline Other & $94,962(47.6)$ & $14,003(68.9)$ & $7556(32.9)$ & \\
\hline Unknown & $4510(54.0)$ & $5028(74.0)$ & 1964 (42.7) & \\
\hline Education & & & & $<0.0001$ \\
\hline$<$ High school & $93,621(60.1)$ & $107,552(67.0)$ & $66,381(61.3)$ & \\
\hline High school & $102,544(59.8)$ & $112,487(69.4)$ & $75,024(55.4)$ & \\
\hline >High school & $208,325(70.1)$ & 180,368 (77.7) & $94,516(56.3)$ & \\
\hline Income & & & & $<0.0001$ \\
\hline$<\$ 25 \mathrm{~K}$ & $169,511(57.6)$ & $178,288(70.2)$ & $102,862(57.8)$ & \\
\hline$\$ 25 \mathrm{~K}-\$ 50 \mathrm{~K}$ & $84,218(76.5)$ & $85,257(74.8)$ & $59,350(64.8)$ & \\
\hline$>\$ 50 \mathrm{~K}$ & $87,213(82.4)$ & $75,320(82.7)$ & $36,810(51.6)$ & \\
\hline Marital status & & & & $<0.0001$ \\
\hline Single relationship & $158,617(59.4)$ & $173,558(70.2)$ & $99,261(54.0)$ & \\
\hline Couple relationship & $245,095(68.7)$ & 225,195 (73.7) & $136,440(59.9)$ & \\
\hline High blood pressure & & & & $<0.0001$ \\
\hline Yes & $344,584(66.4)$ & $327,861(73.9)$ & $192,508(63.0)$ & \\
\hline No & $60,857(58.2)$ & $71,196(65.2)$ & $41,968(41.9)$ & \\
\hline Smoking & & & & $<0.0001$ \\
\hline 1 every day & $42,814(64.5)$ & $54,670(71.7)$ & $30,290(55.1)$ & \\
\hline 2 some days & $14,002(49.2)$ & $17,855(61.9)$ & $16,538(50.6)$ & \\
\hline Not at all & $178,082(72.1)$ & 195,107 (79.5) & $90,360(63.7)$ & \\
\hline Cholesterol & & & & $<0.0001$ \\
\hline Yes & $281,221(69.3)$ & $262,961(75.1)$ & $139,140(58.3)$ & \\
\hline No & $112,368(60.5)$ & $112,913(70.4)$ & $80,942(59.4)$ & \\
\hline Diabetes & & & & $<0.0001$ \\
\hline Yes & $178,506(75.4)$ & $172,219(75.7)$ & $88,971(71.6)$ & \\
\hline No & $210,691(58.3)$ & $212,351(70.0)$ & $136,382(50.8)$ & \\
\hline BMI & & & & $<0.0001$ \\
\hline Underweight & 3525 (31.7) & $14,361(76.8)$ & $2617(61.9)$ & \\
\hline Normal weight & $83,675(66.2)$ & $75,671(67.3)$ & $51,519(46.8)$ & \\
\hline Overweight & $145,449(64.6)$ & $144,373(74.5)$ & $76,820(63.4)$ & \\
\hline Obese & $163,510(67.5)$ & $153,999(71.9)$ & $97,121(61.3)$ & \\
\hline
\end{tabular}


Table 3. Cont.

\begin{tabular}{|c|c|c|c|c|}
\hline \multirow[b]{2}{*}{ Variables } & \multicolumn{3}{|c|}{ Aspirin Use } & \multirow[b]{2}{*}{$p$-Value } \\
\hline & $\begin{array}{l}\text { Angina } \\
N(\%)\end{array}$ & $\begin{array}{c}\text { Myocardial Infarction } \\
\qquad N(\%)\end{array}$ & $\begin{array}{l}\text { Stroke } \\
N(\%)\end{array}$ & \\
\hline General health & & & & $<0.0001$ \\
\hline Poor/Fair & $244,754(62.9)$ & $124,095(76.0)$ & $122,761(60.6)$ & \\
\hline Good/Very good & $146,516(68.7)$ & $34,252(68.1)$ & $97,299(56.3)$ & \\
\hline Excellent & $131,82(60.7)$ & $15,937(56.5)$ & $14,720(41.7)$ & \\
\hline
\end{tabular}

\subsection{Multivariate Logistic Regression Analysis of Factors Associated with Aspirin Use}

Angina, MI, and stroke were grouped together as one class of CVD, and the factors associated with aspirin use were determined from multivariate logistic regression analysis (Table 4). Adjusted odds ratio (aOR), 95\% confidence interval, and $p$-values were calculated. Statistically significant determinants of aspirin use among respondents with CVD included sex, education, high blood pressure, diabetes, and general health. Females and respondents with less than high school education were less likely to use aspirin compared with males and respondents with more than high school education ( $\mathrm{aOR}=0.62 ; p<0.0001$, and $\mathrm{aOR}=0.62 ; p=0.03$, respectively). Also, respondents with CVD who have high blood pressure and diabetes were more likely to use aspirin compared with those without the disorders ( $\mathrm{aOR}=1.46 ; p<0.01$, and $\mathrm{aOR}=1.54 ; p<0.01$, respectively). Those that reported poor/fair general health and good/very good general health were 6.07 times $(p<0.001)$ and 2.77 times $(p<0.01)$ more likely to use aspirin compared with those with excellent general health.

Table 4. Multivariate logistic regression analysis of factors associated with aspirin use among respondents based on CVD status: BRFSS 2015 data.

\begin{tabular}{|c|c|c|c|}
\hline Variables & Odds Ratio & 95\% Confidence Interval & $p$-Value \\
\hline \multicolumn{4}{|l|}{ Sex } \\
\hline Male & Ref. & & \\
\hline Female & 0.62 & $0.49-0.79$ & $<0.0001$ \\
\hline \multicolumn{4}{|l|}{ Age (years) } \\
\hline $18-34$ & Ref. & & \\
\hline $35-44$ & 1.13 & $0.19-6.78$ & 0.89 \\
\hline $45-64$ & 0.84 & $0.17-4.18$ & 0.83 \\
\hline $65+$ & 1.24 & $0.24-6.17$ & 0.79 \\
\hline \multicolumn{4}{|l|}{ Race/Ethnicity } \\
\hline NH white & Ref. & & \\
\hline NH black & 0.99 & $0.62-1.59$ & 0.98 \\
\hline Hispanic & 0.77 & $0.41-1.44$ & 0.42 \\
\hline Other & 0.90 & $0.30-2.65$ & 0.85 \\
\hline \multicolumn{4}{|l|}{ Education } \\
\hline$>$ High school & Ref. & & \\
\hline High school & 0.68 & $0.45-1.03$ & 0.07 \\
\hline$<$ High school & 0.62 & $0.41-0.94$ & 0.03 \\
\hline \multicolumn{4}{|l|}{ Income } \\
\hline$>\$ 50 \mathrm{~K}$ & Ref. & & \\
\hline$\$ 25 \mathrm{~K}-\$ 50 \mathrm{~K}$ & 0.91 & $0.67-1.24$ & 0.55 \\
\hline$<\$ 25 \mathrm{~K}$ & 0.73 & $0.52-1.03$ & 0.07 \\
\hline \multicolumn{4}{|l|}{ Marital status } \\
\hline Single relationship & 0.83 & $0.64-1.08$ & 0.16 \\
\hline Couple relationship & Ref. & & \\
\hline \multicolumn{4}{|l|}{ Insurance } \\
\hline Yes & Ref. & & \\
\hline No & 0.69 & $0.35-1.37$ & 0.29 \\
\hline
\end{tabular}


Table 4. Cont.

\begin{tabular}{lccc}
\hline \multicolumn{1}{c}{ Variables } & Odds Ratio & 95\% Confidence Interval & $p$-Value \\
\hline High blood pressure & Ref. & & \\
No & 1.46 & $1.11-1.93$ & \\
Yes & & & \\
\hline Smoking & Ref. & & 0.21 \\
Not at all & 0.82 & $0.60-1.12$ & 0.92 \\
1 every day & 1.03 & $0.63-1.66$ & \\
2 some days & & & 0.22 \\
\hline Cholesterol & Ref. & & \\
No & 1.17 & $0.91-1.51$ & $<0.01$ \\
Yes & & & \\
\hline Diabetes & Ref. & & \\
No & 1.54 & $1.19-1.99$ & 0.15 \\
Yes & & & 0.14 \\
\hline BMI & Ref. & & 0.07 \\
Underweight & 0.45 & $0.15-1.34$ & $<0.01$ \\
Normal weight & 0.44 & $0.15-1.30$ & $<001$ \\
Overweight & 0.37 & $0.12-1.10$ & \\
Obese & & & \\
\hline General health & Ref. & $3.29-11.18$ & \\
Excellent & 2.77 & & \\
Good/Very good & 6.07 & & \\
Poor/Fair & & & \\
\hline & & & \\
\hline
\end{tabular}

\section{Discussion}

The 2015 BRFSS dataset was analyzed to examine the use of aspirin for the secondary prevention of CVD and the associated factors among adults with CVD in the United States. The prevalence of three forms of CVD (angina pectoris, myocardial infarction, and cerebrovascular disease (stroke)) in a sample of American adults was also determined. Almost $16 \%$ of the study population had angina, MI, or stroke. Weighted percentages of respondents with angina, MI, and stroke were $4 \%, 4.3 \%$, and $3 \%$, respectively. Overall, weighted prevalence of daily (or every other day) aspirin use was about $65 \%, 71 \%$, and $57 \%$ among respondents with angina, MI, and stroke, respectively. Aspirin use among respondents with CVD was associated with male sex, more than high school education, high blood pressure, diabetes, and less than excellent general health.

The results of the current study are similar to those reported by Luepker et al. [28]. Men were more likely to use aspirin than women at any age, and aspirin use was highest in those aged 65 to 74 years. Jing Fang et al. [29] examined the 2013 BRFSS data for the use of aspirin for the secondary prevention of atherosclerotic CVD among adults in 20 states and the District of Columbia in the United States and revealed that $12.5 \%$ of respondents had coronary heart disease, stroke, or both, and about $71 \%$ used aspirin. Almost $94 \%, 80 \%$, and $76 \%$ took aspirin to prevent heart attack, stroke, or both, respectively. Also, aspirin use was more likely among males, $\mathrm{NH}$ white, and 65 years and older. A previous study by Jing Fang et al. [30] using the 2012 National Health Interview Survey also showed that men, 65 years and older, and $\mathrm{NH}$ whites were significantly more likely to take low-dose aspirin for secondary prevention and that hypertension, diabetes, high blood cholesterol, and current smoking were related to low-dose aspirin use. In contrast to the current study, race and smoking status were not significantly associated with aspirin use. Results from Mendy et al. [20] showed that, in Mississippi, United States, $18.9 \%$ of men and $17.8 \%$ of women reported having CVD, and among those with CVD, $85.9 \%$ of men and $85.1 \%$ of women reported taking aspirin. Greater than high school education and diabetes were significantly associated with aspirin use for secondary prevention of CVD among women, while only the white race was the associated factor in men. Analysis of the 
2000-2002 Medical Expenditure Panel Surveys by Opotowsky et al. [31] showed that women (62.4\% vs. $75.6 \%$ men) and those without hypertension ( $66.1 \%$ vs. $72.1 \%$ with hypertension) were less likely to use aspirin regularly, and in contrast to the current study, diabetes was not significantly associated with aspirin use. Stuntz et al. [32] presented results using data from the 2012-2015 National Health Interview Survey and revealed coronary heart disease as the most common CVD (7.1\%), followed by myocardial infarction (5.0\%), stroke (4.1\%), and angina pectoris (3.0\%). Prevalence of aspirin use was highest among males, 65 years and older, NH blacks, living in the Midwest region, with household annual income greater than $\$ 35,000$, with health insurance, obese, and with less than high school education. Body mass index was not associated with aspirin use in the current study.

The current report reveals existing differences based on diagnosis in the use of aspirin for secondary prevention among individuals with CVD. More men reported using aspirin than women. One possible explanation for the gender differences is that women are more likely to report contraindications to aspirin use than men [31-34]. Gender differences in aspirin use could also be explained by uncertainty and related biases among treating physicians, since providers may generalize the unclear efficacy of aspirin in primary prevention for women at low risk of vascular events to women with known CVD [33,34]. The prevalence of aspirin use was also highest among those with MI and least for those with stroke. Hemorrhagic stroke may have been a contraindication for aspirin use, hence the lower prevalence among respondents with stroke. Compared to individuals with higher educational attainment, those with less than high school education had a much higher proportion of CVD but lesser proportion of aspirin utilization. The reason for this observation is worth further investigation. Could this be taken as evidence for the cardioprotective effect of aspirin? Or is lower educational (and social) status a barrier to aspirin access?

The role of aspirin for individuals without CVD is more controversial [35], and the risk of bleeding complications from aspirin ingestion has called into question the level of baseline CVD risk for which use of aspirin in primary prevention is clinically acceptable [36]. However, more than $84 \%$ of the current study population did not report having any form of CVD and less than $32 \%$ took aspirin for primary prevention (results not shown). Mendy et al. [20] also observed that, among those without CVD, 39.1\% of men and $45.9 \%$ of women reported taking aspirin. These results probably reflect the controversy surrounding aspirin use for primary prevention of CVD.

There are limitations to the interpretation of data from the current study. Only three forms of CVD were examined for prevalence of aspirin use. The BRFSS data did not include other forms of CVD, such as peripheral arterial disease and deep venous thrombosis, and therefore these results may not be generalized to include these cases. Also, the questions for identifying persons with CVD (e.g., "Have you ever been told by a doctor, nurse, or other health professional that you had a heart attack, also called a myocardial infarction?") did not include individuals with asymptomatic CVD, without previous thrombotic episodes. Errors in the true estimates of aspirin use among women and respondents with stroke may have occurred as a result of contraindications to aspirin use that were not factored into the analyses. It was not possible to differentiate between thromboembolic and hemorrhagic stroke in the BRFSS data that was used for this study. This may partially account for the lower reported prevalence of aspirin use for secondary prevention of stroke compared with heart attack. Limiting the analysis to those without a contraindication to aspirin use may narrow the absolute gender gap [31]. Recall bias from self-reporting may have caused overestimation, underestimation, or misclassification of the results presented. Despite these limitations, data from the BRFSS are reliable and generally valid because the content of the survey questions, questionnaire design, data collection, procedures, interviewing techniques, and data processing have been developed to improve data quality [37]. Data from this study can be helpful to clinicians and public health practitioners in directing resources for promoting aspirin use toward groups with lower prevalence. 


\section{Conclusions}

Regular use of aspirin for secondary prevention of CVD has been proven to cause a significant reduction in morbidity and mortality attributed to CVD. However, not all groups of individuals are leveraging the benefits from the recommended use of aspirin and may consequently be at higher risks for cardiovascular events and premature death. Further research is needed to better understand the underlying causes of lower rates of aspirin use among identified individuals with CVD. Strategies to increase aspirin use may include making policies that promote utilization at all levels of primary and public health care, educating patients and caregivers about the benefits of aspirin, and implementing interventions that target the underserved.

Author Contributions: Conceptualization, B.E.A.; methodology, B.E.A., Z.H., and N.L.; software, B.E.A. and Z.H.; validation, B.E.A.; formal analysis, B.E.A. and Z.H.; data curation, Z.H. and B.E.A.; writing-original draft preparation, B.E.A.; writing-review and editing, N.L., C.S., A.H., R.S., and T.J.

Acknowledgments: We thank the Augusta University IPPH 2018 Summer Public Health Scholars Program for supporting Zachary Hoffman and Nicollette Lewis during the course of this project.

Conflicts of Interest: The authors declare no conflict of interest.

\section{References}

1. WHO Cardiovascular Diseases (CVDs). Available online: http://www.who.int/mediacentre/factsheets/ fs317/en/ (accessed on 7 August 2018).

2. Mendis, S.; Puska, P.; Norrving, B. (Eds.) Global Atlas on Cardiovascular Disease Prevention and Control; World Health Organization: Geneva, Switzerland, 2011.

3. Stewart, J.; Manmathan, G.; Wilkinson, P. Primary prevention of cardiovascular disease: A review of contemporary guidance and literature. JRSM Cardiovasc. Dis. 2017, 6. [CrossRef] [PubMed]

4. Benjamin, E.J.; Blaha, M.J.; Chiuve, S.E.; Cushman, M.; Das, S.R.; Deo, R.; de Ferranti, S.D.; Floyd, J.; Fornage, M.; Gillespie, C.; et al. On behalf of the American Heart Association Statistics Committee and Stroke Statistics Subcommittee. Heart disease and stroke statistics-2017 update: A report from the American Heart Association [published online ahead of print January 25, 2017]. Circulation 2013. [CrossRef]

5. Piepoli, M.F.; Hoes, A.W.; Agewall, S.; Albus, C.; Brotons, C.; Catapano, A.L.; Cooney, M.T.; Corrà, U.; Cosyns, B.; Deaton, C.; et al. European Guidelines on cardiovascular disease prevention in clinical practice: The Sixth Joint Task Force of the European Society of Cardiology and Other Societies on Cardiovascular Disease Prevention in Clinical Practice (constituted by representatives of 10 societies and by invited experts) Developed with the special contribution of the European Association for Cardiovascular Prevention \& Rehabilitation (EACPR). Eur. Heart J. 2016, 37, 2315-2381. [PubMed]

6. Eckel, R.H.; Jakicic, J.M.; Ard, J.D.; De Jesus, J.M.; Miller, N.H.; Hubbard, V.S.; Lee, I.M.; Lichtenstein, A.H.; Loria, C.M.; Millen, B.E.; et al. AHA/ACC guideline on lifestyle management to reduce cardiovascular risk: A report of the American College of Cardiology / American Heart Association Task Force on Practice Guidelines. J. Am. Coll. Cardiol. 2013, 2014, 63.

7. Miner, J.; Hoffhines, A. The discovery of aspirin's antithrombotic effects. Tex. Heart Inst. J. 2007, 34, 179-186. [PubMed]

8. Ittaman, S.V.; VanWormer, J.J.; Rezkalla, S.H. The role of aspirin in the prevention of cardiovascular disease. Clin. Med. Res. 2014, 12, 147-154. [CrossRef] [PubMed]

9. Vane, J.R.; Botting, R.M. The mechanism of action of aspirin. Thromb. Res. 2003, 110, 255-258. [CrossRef]

10. Warner, T.D.; Nylander, S.; Whatling, C. Anti-platelet therapy: Cyclo-oxygenase inhibition and the use of aspirin with particular regard to dual anti-platelet therapy. Br. J. Clin. Pharmaco. 2011, 72, 619-633. [CrossRef]

11. Dippel, D.W.; Van Kooten, F.; Leebeek, F.W.; van Vilet, H.H.; Mehicevic, A.; Li, S.S.; Koudstaal, P.J. What is the lowest dose of aspirin for maximum suppression of in vivo thromboxane production after a transient ischemic attack or ischemic stroke? Cerebrovasc. Dis. 2004, 17, 296-302. [CrossRef]

12. Final Recommendation Statement: Aspirin Use to Prevent Cardiovascular Disease and Colorectal Cancer: Preventive Medication. U.S. Preventive Services Task Force. September 2017. Available online: https://www.uspreventiveservicestaskforce.org/Page/Document/RecommendationStatementFinal/ aspirin-to-prevent-cardiovascular-disease-and-cancer (accessed on 7 August 2018). 
13. Guirguis-Blake, J.M.; Evans, C.V.; Senger, C.A.; Rowland, M.G.; O'Connor, E.A.; Whitlock, E.P. Aspirin for the Primary Prevention of Cardiovascular Events: A Systematic Evidence Review for the U.S. Preventive Services Task Force. Evidence Synthesis No. 131; AHRQ Publication No. 13-05195-EF-1; Agency for Healthcare Research and Quality: Rockville, MD, USA, 2015.

14. American Heart Association. Aspirin and Heart Disease. Available online: http://www.heart.org/ HEARTORG/Conditions/HeartAttack/PreventionTreatmentofHeartAttack/Aspirin-and-Heart-Disease_ UCM_321714_Article.jsp\#.W2oJHMIpC70 (accessed on 7 August 2018).

15. Bell, A.D.; Roussin, A.; Cartier, R.; Chan, W.S.; Douketis, J.D.; Gupta, A.; Kraw, M.E.; Lindsay, T.F.; Love, M.P.; Pannu, N.; et al. The use of antiplatelet therapy in the outpatient setting: Canadian Cardiovascular Society Guidelines Executive Summary. Can. J. Cardiol. 2011, 27, 208221. [CrossRef]

16. Graham, I.; Atar, D.; Borch-Johnson, K.; Boysen, G.; Burell, G.; Cifkova, R.; Dallongeville, J.; De Backer, G.; Ebrahim, S.; Gjelsvik, B.; et al. ESC Committee for Practice Guidelines. European guidelines on cardiovascular disease prevention in clinical practice: Executive summary. Atherosclerosis 2007, 194, 1-45. [CrossRef] [PubMed]

17. ISIS-2 (Second International Study of Infarct Survival) Collaborative Group. Randomised trial of intravenous streptokinase, oral aspirin, both, or neither among 17,187 cases of suspected acute myocardial infarction: ISIS-2. Lancet 1988, 2, 349-360.

18. Godley, R.W.; Hernandez-Vila, E. Aspirin for Primary and Secondary Prevention of Cardiovascular Disease. Tex. Heart Inst. J. 2016, 43, 318-319. [CrossRef] [PubMed]

19. Centers for Disease Control and Prevention, Behavioral Risk Factor Surveillance System (BRFSS). About BRFSS. Available online: http:/ / www.cdc.gov/brfss/about/index.htm (accessed on 8 August 2018).

20. Mendy, V.L.; Vargas, R.; Zhang, L. Aspirin for Prevention of Cardiovascular Disease. Prev. Chronic. Dis. 2017, 14, E77. [CrossRef] [PubMed]

21. Ansa, B.E.; White, S.; Chung, Y.; Smith, S.A. Trends in HIV Testing among Adults in Georgia: Analysis of the 2011-2015 BRFSS Data. Int. J. Environ. Res. Public Health 2016, 13, 1126. [CrossRef] [PubMed]

22. American Association for Public Opinion Research. Standard Definitions. Available online: https:// www.aapor.org/AAPOR_Main/media/publications/Standard-Definitions2015_8theditionwithchanges_ April2015_logo.pdf (accessed on 25 October 2016).

23. Centers for Disease Control and Prevention. Behavioral Risk Factor Surveillance System. 2015 Summary Data Quality Report. (29 July 2015). Available online: http:/ / www.cdc.gov/brfss/annual_data/2015/pdf/ 2015-sdqr.pdf (accessed on 25 October 2016).

24. Centers for Disease Control and Prevention. Behavioral Risk Factor Surveillance System. Weighting BRFSS Data BRFSS 2014. Available online: https://www.cdc.gov/brfss/annual_data/2014/pdf/weighting-data. pdf (accessed on 25 October 2016).

25. The Complete Guide to Market Research. Data Preparation. Available online: https://www. qresearchsoftware.com/market-research-guide-data-preparation (accessed on 7 February 2019).

26. IBM Corp. Released 2017. IBM SPSS Statistics for Windows, Version 25.0; IBM Corp: Armonk, NY, USA, 2017.

27. Bernstein, A.B.; Sweeney, M.H. Centers for Disease Control and Prevention. Public health surveillance data: Legal, policy, ethical, regulatory, and practical issues. MMWR Suppl. 2012, 61, 30-34. [PubMed]

28. Luepker, R.V.; Steffen, L.M.; Duval, S.; Zantek, N.D.; Zhou, X.; Hirsch, A.T. Population Trends in Aspirin Use for Cardiovascular Disease Prevention 1980-2009: The Minnesota Heart Survey. J. Am. Heart Assoc. 2015, 4, e002320. [CrossRef]

29. Fang, J.; George, M.G.; Hong, Y.; Loustalot, F. Use of Aspirin for Prevention of Recurrent Atherosclerotic Cardiovascular Disease Among Adults-20 States and the District of Columbia, 2013. MMWR Morb. Mortal. Wkly. Rep. 2015, 64, 733-737.

30. Fang, J.; George, M.G.; Gindi, R.M.; Hong, Y.; Yang, Q.; Ayala, C.; Ward, B.W.; Loustalot, F. Use of low-dose aspirin as secondary prevention of atherosclerotic cardiovascular disease in US adults (from the National Health Interview Survey, 2012). Am. J. Cardiol. 2015, 115, 895-900. [CrossRef]

31. Opotowsky, A.R.; McWilliams, J.M.; Cannon, C.P. Gender differences in aspirin use among adults with coronary heart disease in the United States. J. Gen. Intern. Med. 2007, 22, 55-61. [CrossRef]

32. Stuntz, M.; Bernstein, B. Recent trends in the prevalence of low-dose aspirin use for primary and secondary prevention of cardiovascular disease in the United States, 2012-2015. Prev. Med. Rep. 2017, 5, 183-186. [CrossRef] [PubMed] 
33. Berger, J.S.; Roncaglioni, M.C.; Avanzini, F.; Pangrazzi, I.; Tognoni, G.; Brown, D.L. Aspirin for the primary prevention of cardiovascular events in women and men: A sex-specific meta-analysis of randomized controlled trials. JAMA 2006, 295, 306-313. [CrossRef] [PubMed]

34. Mulrow, C.; Pignone, M. An editorial update: Should she take aspirin? Ann. Intern. Med. 2005, 142, 942-943. [CrossRef] [PubMed]

35. Patrono, C.; Rodriguez, L.A.G.; Landolfi, R.; Baigent, C. Drug therapy-Low-dose aspirin for the prevention of atherothrombosis. N. Eng. J. Med. 2005, 353, 2373-2383. [CrossRef] [PubMed]

36. Capodanno, D.; Angiolillo, D.J. Aspirin for primary prevention of cardiovascular disease. Lancet 2018, 392, 988-990. [CrossRef]

37. Barrett-Connor, E.; Ayanian, J.Z.; Brown, E.R.; Coultas, D.B.; Francis, C.K.; Goldberg, R.J.; Gostin, L.O.; Kottke, T.E.; Lee, E.T.; Mannino, D.M. A Nationwide Framework for Surveillance of Cardiovascular and Chronic Lung Diseases; National Academies Press (U.S.): Washington, DC, USA, 2011.

2019 by the authors. Licensee MDPI, Basel, Switzerland. This article is an open access article distributed under the terms and conditions of the Creative Commons Attribution (CC BY) license (http:// creativecommons.org/licenses/by/4.0/). 